\title{
Phylogenetic footprint of the plant clock system in angiosperms: evolutionary processes of Pseudo-Response Regulators
}

\author{
Naoki Takata1,4, Shigeru Saito 2,5, Claire Tanaka Saito 2 and Matsuo Uemura*1,3
}

\begin{abstract}
Background: Plant circadian clocks regulate many photoperiodic and diurnal responses that are conserved among plant species. The plant circadian clock system has been uncovered in the model plant, Arabidopsis thaliana, using genetics and systems biology approaches. However, it is still not clear how the clock system had been organized in the evolutionary history of plants. We recently revealed the molecular phylogeny of LHY/CCA1 genes, one of the essential components of the clock system. The aims of this study are to reconstruct the phylogenetic relationships of angiosperm clock-associated PRR genes, the partner of the LHY/CCA1 genes, and to clarify the evolutionary history of the plant clock system in angiosperm lineages.

Results: In the present study, to investigate the molecular phylogeny of PRR genes, we performed two approaches: reconstruction of phylogenetic trees and examination of syntenic relationships. Phylogenetic analyses revealed that PRR genes had diverged into three clades prior to the speciation of monocots and eudicots. Furthermore, copy numbers of $P R R$ genes have been independently increased in monocots and eudicots as a result of ancient chromosomal duplication events.

Conclusions: Based on the molecular phylogenies of both PRR genes and LHY/CCA1 genes, we inferred the evolutionary process of the plant clock system in angiosperms. This scenario provides evolutionary information that a common ancestor of monocots and eudicots had retained the basic components required for reconstructing a clock system and that the plant circadian clock may have become a more elaborate mechanism after the speciation of monocots and eudicots because of the gene expansion that resulted from polyploidy events.
\end{abstract}

\section{Background}

Many organisms such as cyanobacteria, fruit flies, mammals and plants have an endogenous time-keeping mechanism, a circadian clock, to gauge daily and seasonal environmental changes. Circadian clock systems in plants regulate various photoperiodic and diurnal responses, such as photomorphogenic processes, floral transition, leaf movements, stomatal conductance, photosynthetic capacity, and volatile emissions (reviewed in [1]). Among these, means to discriminate the length of the photoperiod are conserved among plant species, and it is commonly thought that circadian clock system of plants

\footnotetext{
*Correspondence: uemura@iwate-u.ac.jp

1 United Graduate School of Agricultural Sciences, Iwate University, Morioka 020-8550, Japan

Full list of author information is available at the end of the article
}

shares a basic mechanism that controls photoperiodic responses.

In the past decade, numerous molecular genetic analyses of the model plant Arabidopsis thaliana have uncovered the basic molecular network of the plant circadian clock (reviewed in [2,3]). Mathematical analyses have been used to develop a computational model of the plant clock system, which contains the main transcriptional feedback loop (Loop I) and two additional loops (Loops II and III) associated with the main loop (Figure 1) $[4,5]$. This multiple feedback loop system of the plant clock system is composed of two gene families, Pseudo-Response Regulators (PRRs) and Late Elongated Hypocotyl/Circadian Clock Associated 1 (LHY/CCA1), and two unknown factors ("X" and "Y"). The main feedback loop (Loop I) consists of two LHY/CCA1 genes, the Pseudo-Response Regulator 1/Timing of CAB2 Expression 1 (PRR1/TOC1) 


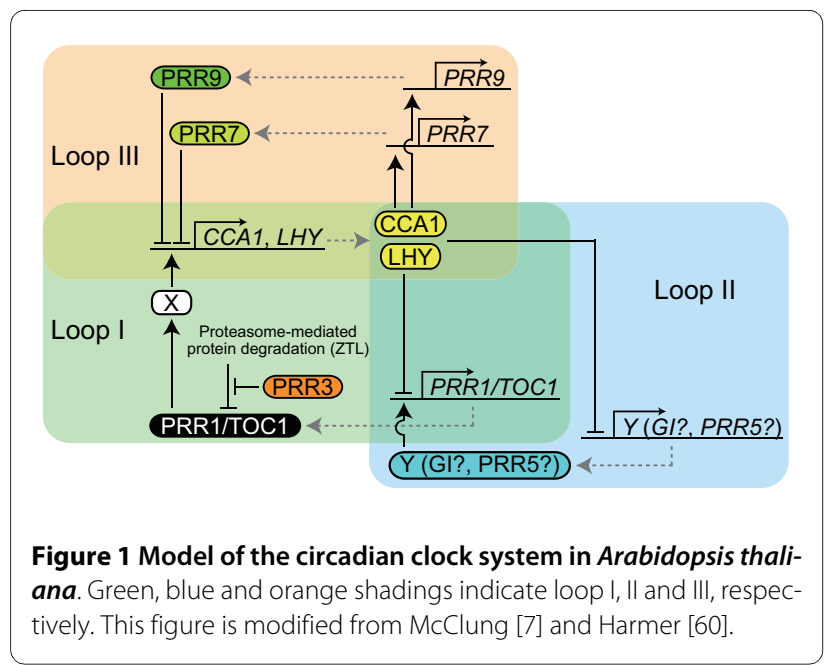

gene and the unknown factor "X". In this loop, the feedback regulatory network operates as follows: the eveningacting PRR1/TOC1 gene induces the morning-acting $L H Y$ and $C C A 1$ genes via the unknown factor " $\mathrm{X}$ ", and is in turn repressed by $L H Y / C C A 1$ [6]. The Loop I associates with Loop II via the PRR1/TOC1 gene and with Loop III via $L H Y / C C A 1$ genes $[4,5]$. Loop II is made up of PRR1/TOC1 and an unknown factor "Y". It has been proposed that the unknown factor "Y" is GIGANTEA and/or $P R R 5$, although the true component has not yet been identified [7]. Loop III consists of $L H Y / C C A 1$ genes and two PRR genes, $P R R 7$ and PRR9. Together, the gene families PRRs and $L H Y / C C A 1 \mathrm{~s}$ have key roles and form the complex regulatory network in the plant clock system.

Clock-associated $P R R$ genes are conserved among angiosperm evolutionary lineages as are their partner $L H Y / C C A 1$ genes $[8,9]$. In eudicotyledonous plants, five copies of $P R R$ genes have been identified in A. thaliana and Carica papaya and seven copies have been found in Populus trichocarpa [10-12]. In monocotyledonous plants, Oryza sativa has five PRR genes [13]. The expression patterns of $P R R$ genes in A. thaliana and O. sativa share some common features. The five $P R R$ genes in $A$. thaliana show diurnal and sequential-temporal expression patterns from dawn to dusk as follows; PRR9TPRR7TPRR5TPRR3TPRR1 [10]. The similar sequential expression pattern is found in homologous genes of $O$. sativa, which are expressed as follows; OsPRR73 (OsPRR37)TOsPRR95 (OsPRR59)TOsPRR1 [13]. In spite of these similarities in the copy numbers and the expression patterns of clock-associated $P R R$ genes, it is still unclear how the $P R R$ genes have evolved in monocots and eudicots and how they have been incorporated in the regulatory network of the clock system in the evolutionary history of plants.

Rapid accumulation of genomic sequence data offers new perspectives on the molecular phylogeny of genes in angiosperms [14]. Completion of genomic sequences for various plant species reveals that angiosperm genomes have undergone several ancient chromosomal or whole genome duplication events [11,15-17]. In monocot lineages, the $\rho$ polyploidy event occurred before the speciation of O. sativa and Sorghum bicolor in commelinids $[18,19]$. On the other hand, four polyploidy events appear to have occurred in eudicot lineages. Among these polyploidy events, the $\gamma$ triplication event took place near the base of the eudicot clade though the timing of this event is still being debated $[11,14,16,17]$. The draft genomic sequence analysis of $C$. papaya has revealed that the genome of $A$. thaliana underwent two polyploidy events ( $\alpha$ and $\beta$ ) after the speciation of C. papaya and A. thaliana in eurosids II [11]. Furthermore, the $\beta$ polyploidy event is thought to have occurred before the $\alpha$ event [14]. In eurosids I, the salicoid polyploidy event occurred within the Salicaceae lineages, which includes Populus [17]. The footprints of these chromosomal duplication events are the conserved order of the genes on the duplicated chromosomes in the present genomic sequences [20]. Thus, comparison of the order of genes surrounding duplicated genes provides molecular evolutionary information on their phylogenetic relationships [21,22].

In the present study, to clarify the phylogenetic relationships among angiosperm $P R R$ genes, we (1) identified $P R R$ genes using available genomic databases of eudicots (Vitis vinifera, P. trichocarpa, C. papaya, and A. thaliana) and monocots (O. sativa and S. bicolor) and (2) examined the evolutionary processes of angiosperm $P R R$ genes by conventional phylogenetic reconstruction and examination of syntenic relationships. With these results, we reconstructed the molecular phylogeny of $P R R$ genes in angiosperms and found that gene expansion of PRRs occurred via polyploidy events in monocots and eudicots. Taken together with the molecular phylogeny of the other major gene family of the plant clock system (LHY) CCA1s) [9], our data allow us to explore the evolutionary history of the multiple feedback loop system in angiosperm lineages.

\section{Results}

Identification of clock-associated PRR genes in angiosperms

There are five copies of the PRR genes in the genomes of $O$. sativa, S. bicolor, V. vinifera and C. papaya, six copies in A. thaliana, and eight copies in P. trichocarpa (see Additional files 1 and 2). The PRR1/TOC1 gene in $C$. papaya was not retrieved from the genomic sequence database because the nucleotide sequence of the C-terminal region of the gene has not yet been determined. The angiosperm $P R R$ genes retained a highly conserved PRdomain at the $\mathrm{N}$-terminus and a CCT-motif at the C-terminus (see Additional file 3). However, two PRR-like 
genes (PRR9b in A. thaliana, AtPRR9b, and PRR5c in $P$. trichocarpa, PtPRR5c) retained the CCT-motif but not the PR-domain (see Additional file 4). Thus, we examined the molecular phylogeny of $P R R$ genes but excluded $A t P R R 9 b$ and PtPRR5 $c$ in the present study.

\section{Phylogenetic analysis of PRR gene family}

To deduce the evolutionary relationships among PRR genes in angiosperms, a phylogenetic tree was reconstructed using the minimum evolution (ME) method. Angiosperm $P R R$ genes clearly separated into three clades (PRR1/TOC1 clade, PRR3 and 7 clade, and PRR5 and 9 clade) (Figure 2). This classification was consistent with the categorization of genomic structures of $P R R$ genes that was apparent when exon-intron structures and insertions/deletions variation were examined (Figure 3, see Additional file 5). We found that each clade contains genes from both eudicots and monocots, suggesting that ancient $P R R$ gene(s) diverged into three clades before the speciation of monocots and eudicots.

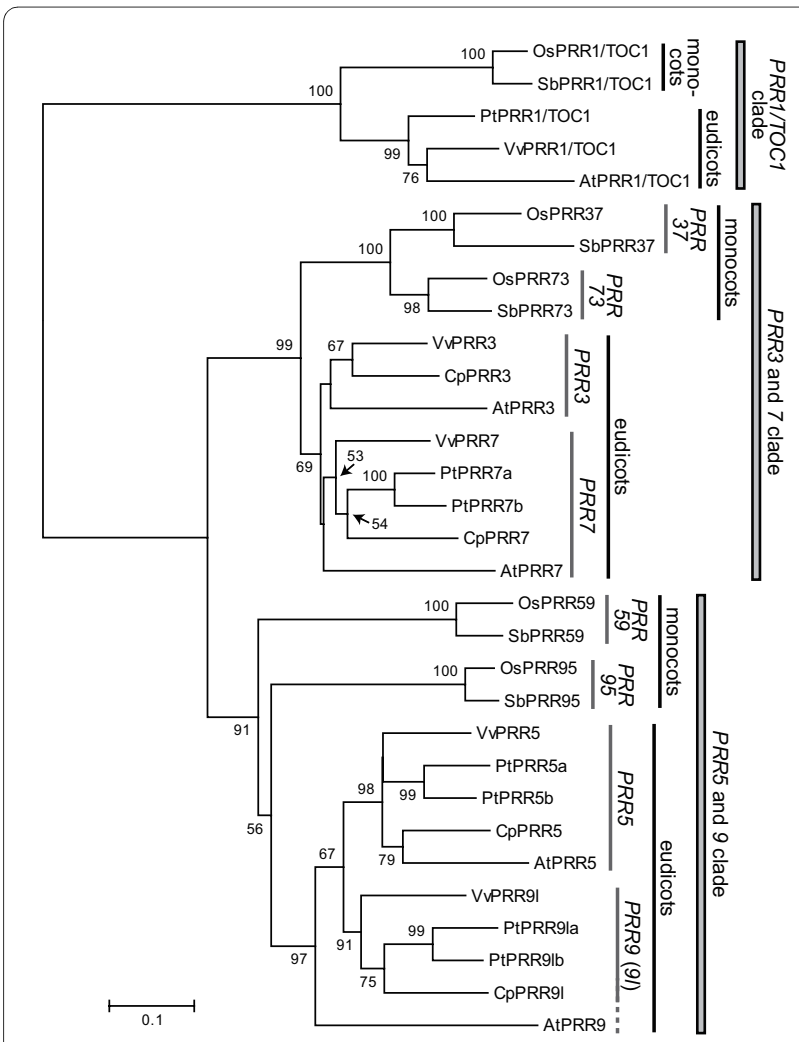

Figure 2 Phylogenetic tree of angiosperm PRR genes. Amino acid sequences were aligned using TCoffee program [47]. The phylogenetic tree was reconstructed by the ME method from the numbers of amino acid substitutions estimated by the JTT model. PRR1/TOC1 genes were utilized as an outgroup in the phylogenetic trees. The numerals at the branch indicate bootstrap values calculated by the ME method with 1,000 replications. Bootstrap values $>50 \%$ are shown
In all plant species examined, one copy of the PRR1/ $T O C 1$ gene was retained in the PRR1/TOC1 clade (Figure 2 ), whereas at least two copies were found in the $P R R 3$ and 7 clade and the PRR 5 and 9 clade. PRR1/TOC1 genes diverged into two clusters representing monocots and eudicots.

The PRR3 and 7 clade consisted of two different clusters, each of which exclusively consists of monocot or eudicot genes (Figure 2). Accordingly, the phylogenetic tree suggested that the gene duplication events producing monocotyledonous PRR37 and PRR73 or eudicotyledonous $P R R 3$ and PRR7 occurred independently within monocot and eudicot lineages, respectively. After the gene duplication event in eudicots, orthologs of $P$. trichocarpa PRR3 appeared to be lost, whereas the $P$. trichocarpa PRR7 gene was duplicated into PRR7a and $7 b$.

In the PRR5 and 9 clade, the monocot PRR59 and PRR95 genes showed an earlier gene duplication that may have occurred in a common ancestor of monocots and eudicots (Figure 2). However, the bootstrap value supporting this branch was not very high, $56 \%$. Eudicotyledonous PRR5 and PRR9/9-like (9l) genes formed a cluster in the phylogenetic tree. In this cluster, A. thaliana PRR was distantly related to other PRR5 and PRR $9 l$ genes, which was also observed in the phylogenetic trees reconstructed by the neighbor-joining, maximum likelihood and Bayesian methods (see Additional files 6 and 7). This topology within PRR5 and 9 clade might be the artefact caused by faster substitution rate of the AtPRR9 gene. Otherwise, sparse taxonomic sampling obscures the additional gene duplication and loss events occurred in eudicots. PRR5a in P. trichocarpa was more closely related to $P R R 5 b$ than other $P R R 5$, and similar close relationship was found between PRR9la and PRR9lb in P. trichocarpa (Figure 2). These findings indicated that the gene duplication events that produced PRR5 $a$ and $5 b$ and PRR9la and $9 l b$ occurred within rosids. Collectively, although the $P R R 3$ and 7 clade and the $P R R 5$ and 9 clade contained at least two copies of $P R R$ genes in both monocots and eudicots, $P R R \mathrm{~s}$ in the two clades are assumed to have independently duplicated in monocot and eudicot lineages.

\section{Functional divergence among PRR gene clusters}

Clock associated-PRR genes were divided into the three gene clusters (PRR1/TOC1 clade, PRR3 and 7 clade, and $P R R 5$ and 9 clade) that had been formed prior to the speciation of monocots and eudicots. Although the amino acid sequences of the genes were highly conserved in the PR-domain and CCT-motif, there were several amino acid changes that were distinctive among the three clades, which potentially contribute to functional differences (see Additional file 3). To detect amino acid substi- 


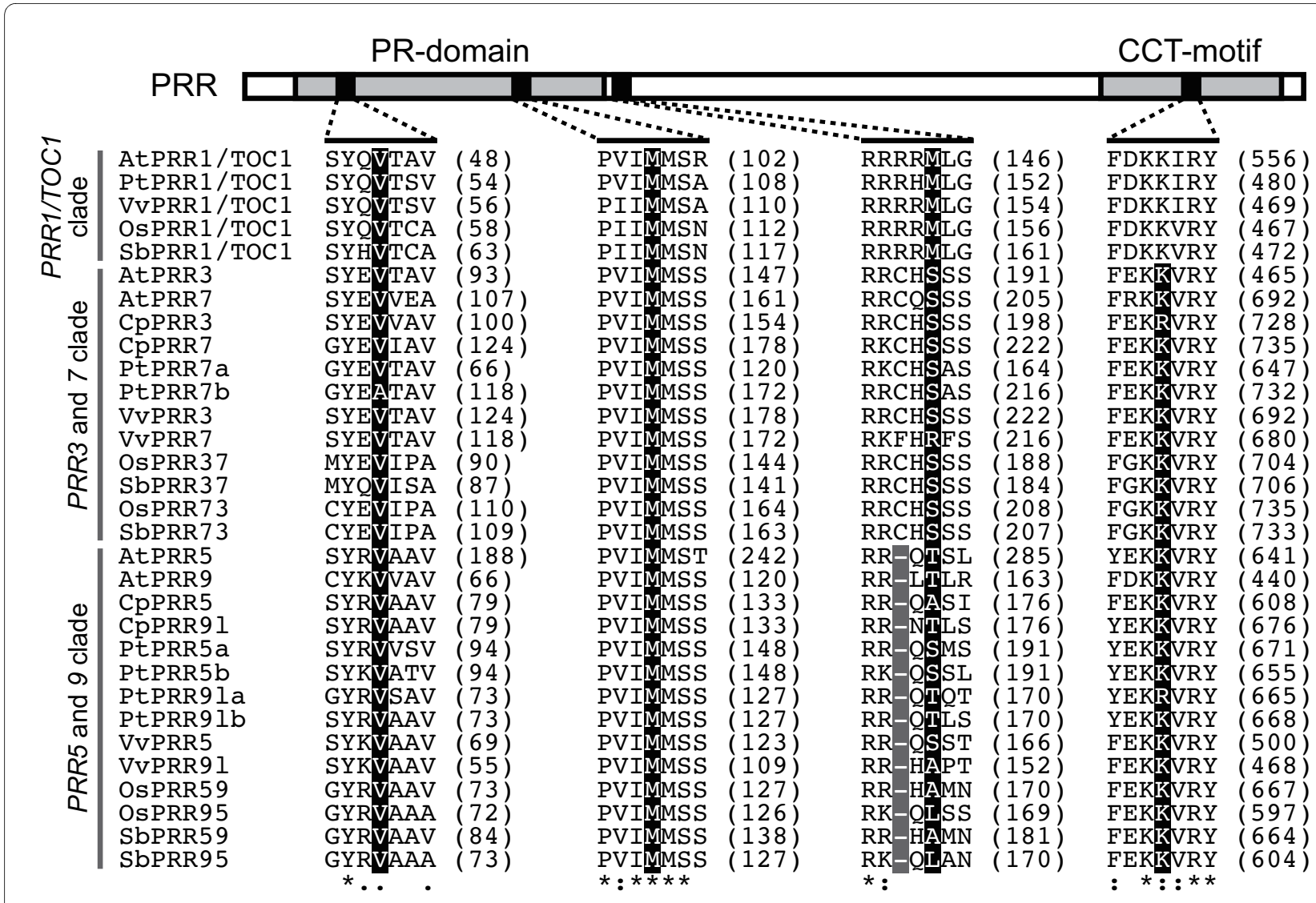

Figure 3 Comparison of the exon-intron structures of $P R R$ genes around the region of PR-domain and CCT-motif. The amino acid sequences encoded by PRR genes were aligned using TCoffee program [47]. Accession numbers and gene IDs of the PRR genes are shown in Additional file 1. The numerals on the right side indicate the numbers of amino acid residues of each protein. Sequence similarity is indicated below the alignment using the symbols "asterisk," "colon," and "dot" for identical, highly similar, and weakly similar residues, respectively. Black and gray shadings on the alignments indicate a site of exon-intron boundary and one-amino acid deletion, respectively.

tutions that are potentially involved in functional divergence among the clades in PR-domain, CCT-motif and their flanking regions, we performed statistical analysis to estimate the coefficient of type I and type II functional divergences $\left(\theta_{\mathrm{I}}\right.$ and $\left.\theta_{\mathrm{II}}\right)$. In the type I functional divergence, sites are conserved in one gene cluster but variable in the sister clusters [23]. On the other hand, type II sites are fixed in both clusters but the amino acid residues are different between the clusters [24]. In the comparison among the PRR gene clusters, all of the coefficients for the type I functional divergence $\left(\theta_{\mathrm{I}}\right)$ were significantly larger than zero (Table 1 ). In addition, the values of the type II functional divergence $\left(\theta_{\mathrm{II}}\right)$ between PRR1/TOC1 clade and PRR3 and 7 clade and between PRR1/TOC1 clade and PRR5 and 9 clade were significantly different from zero while the value between $P R R 3$ and 7 clade and PRR5 and 9 clade was not significantly greater than zero. Six sites that were above the empirical cutoff values were identified in the comparison between
PRR1/TOC1 clade and PRR3 and 7 clade, ten sites in PRR1/TOC1 clade and PRR5 and 9 clade and two sites in $P R R 3$ and 7 clade and PRR5 and 9 clade (Figures 4 and 5). Intriguingly, these sites were predominantly detected in the PR-domain and its flanking region rather than the CCT-motif (Figure 4). These results imply that the amino acid substitutions in the PR-domain, through which PRR proteins interact with other proteins (ZEITLUPE and PRRs) $[25,26]$, may partially contribute to the functional divergence among the three gene clusters.

\section{Phylogenetic relationships of PRR gene family inferred from chromosome syntenies}

To clarify evolutionary events such as gene duplication and gene deletion among angiosperm PRR genes, we investigated chromosomal syntenies among the genomes of monocots or eudicots. Because ancient chromosome duplication events result in conserved gene order on the duplicated chromosomes [20], comparisons of gene organization and detection of chromosomal syntenies can 
Table 1: Coefficients of type I and type II functional divergences among the PRR gene clades.

\begin{tabular}{|c|c|c|c|c|}
\hline \multirow[t]{2}{*}{ Comparison } & \multicolumn{2}{|c|}{ Type I } & \multicolumn{2}{|c|}{ Type II } \\
\hline & $\theta \mathbf{I}$ & SE & өII & SE \\
\hline $\begin{array}{l}\text { PRR1/TOC1 vs PRR3 } \\
\text { and } 7\end{array}$ & 0.366 & $0.109^{*}$ & 0.196 & $0.093^{* *}$ \\
\hline $\begin{array}{l}\text { PRR1/TOC1 vs PRR5 } \\
\text { and } 9\end{array}$ & 0.473 & $0.075^{*}$ & 0.175 & $0.088^{* *}$ \\
\hline $\begin{array}{l}\text { PRR3 and } 7 \text { vs PRR5 } \\
\text { and } 9\end{array}$ & 0.234 & $0.048^{*}$ & -0.003 & 0.106 \\
\hline
\end{tabular}

provide molecular evolutionary information to understand the phylogenetic relationships of the genes [21,22].

In eudicots, the flanking region of PRR1/TOC1, PRR3, 5, 7 and 9 in $A$. thaliana showed a syntenic relationship with PRR1/TOC1, PRR3, 5, 7 and $9 l$ in $V$. vinifera, $P$. trichocarpa, and C. papaya, respectively (Figure 6). In addition, the syntenies were also found in the flanking regions between AtPRR3 and VvPRR7, AtPRR7 and $V v P R R 3, A t P R R 5$ and $V v P R R 9 l$ and AtPRR9 and $V v P R R 5$ (data not shown). The former syntenic relationships between AtPRR3 and VvPRR3, AtPRR7 and VvPRR7, $A t P R R 5$ and $V v P R R 5$ and AtPRR9 and $V v P R R 9 l$ were more conserved than the latter relationships between AtPRR3 and VvPRR7, AtPRR7 and VvPRR3, AtPRR5 and $V v P R R 9 l$ and $A t P R R 9$ and $V v P R R 5$, respectively. Syntenic relationships were not found between the neighbouring regions of $P$. trichocarpa $P R R 1 / T O C 1$ and those of other $P R R 1 / T O C 1 \mathrm{~s}$, or between the neighbouring region of $C$. papaya PRR9l and those of other PRR9/9ls. It is not clear whether these observations can be ascribed to chromosomal rearrangements or fragmentations of genomic sequences in small contigs.

In the genome of $A$. thaliana, the flanking regions of PRR genes showed syntenies with one or three partial regions of its genome (Figure 6). These syntenic relationships originated from the chromosomal duplications that arose from the $\beta$ and $\alpha$ polyploidy events $[27,28]$. In the $P$. trichocarpa genome, two copies of each PRR5, PRR7, and $P R R 9 l$ gene were located at the syntenic regions of chromosomes 12 and 15 (Figure 6B), those of chromosomes 8 and 10 (Figure 6D), and those of chromosomes 2 and 14 (Figure 6E), respectively. Tuskan et al., [17] showed that these syntenic regions were produced via the salicoid polyploidy event. Although flanking region of $A$. thaliana PRR3 shares syntenic relationships with partial regions of $P$. trichocarpa chromosomes 1 and 9, these two partial regions did not retain a $P R R$ gene (Figure $6 C$ ).

Next, we investigated the chromosomal syntenic relationships derived from the $\gamma$ triplication event using the genomic information of $V$. vinifera [16]. There were chromosomal syntenies conserved between the flanking regions of $V v P R R 3$ and $V v P R R 7$ and between the flanking regions of $V v P R R 5$ and $V v P R R 9 l$, which were originated from the $\gamma$ triplication event ([16], see also the comparative genomic tool, CoGe [29]). The synteny of PRR3, 5, 7 and $9 l$ in $V$. vinifera and homologous genes in other eudicotyledonous plants (A. thaliana, P. trichocarpa, and C. papaya), respectively, suggest that the ancestral $P R R 3 /$ 7 gene in eudicots was duplicated into PRR3 and PRR7, and PRR5/9 into PRR5 and PRR9/9l in the $\gamma$ polyploidy event (Figure 7). After the $\gamma$ polyploidy event, one copy of each PRR gene (PRR1/TOC1, PRR3, 5, 7 and 9/9l) has been conserved in the present genomes of $V$. vinifera and C. papaya, which apparently have not undergone additional polyploidy events. Although $A$. thaliana PRR genes were repeatedly duplicated by the $\beta$ and/or $\alpha$ polyploidy events, one copy of each gene remains in the present $A$. thaliana genome, which is similar to $V$. vinifera and $C$. papaya genomes (Figures 6 and 7). In the genome of $P$. trichocarpa, $P R R 5,7$, and $9 l$ were duplicated in the subsequent salicoid polyploidy event, but the PRR3 was lost prior to the salicoid polyploidy event or duplicated PRR3s were lost following the polyploidy event.

The flanking region of the $P R R$ gene in $O$. sativa (OsPRR1/TOC1, OsPRR73, OsPRR59 and OsPRR95) showed conserved synteny with that of orthologous genes in S. bicolor [30]. On the other hand, only a few syntenic regions were identified between PRR37 of $O$. sativa and the orthologous gene of $S$. bicolor. In the genome of $O$. sativa, the neighbouring region of OsPRR37 showed synteny with that of OsPRR73. This syntenic relationship resulted from the chromosomal duplication that occurred in the $\rho$ polyploidy event [18]. The $\rho$ polyploidy event also resulted in conserved chromosomal synteny between the flanking region of OSPRR59 and a partial region of chromosome 8 , and between the flanking region of OSPRR95 and a different partial region of chromosome 8 . However, these partial regions of chromo- 

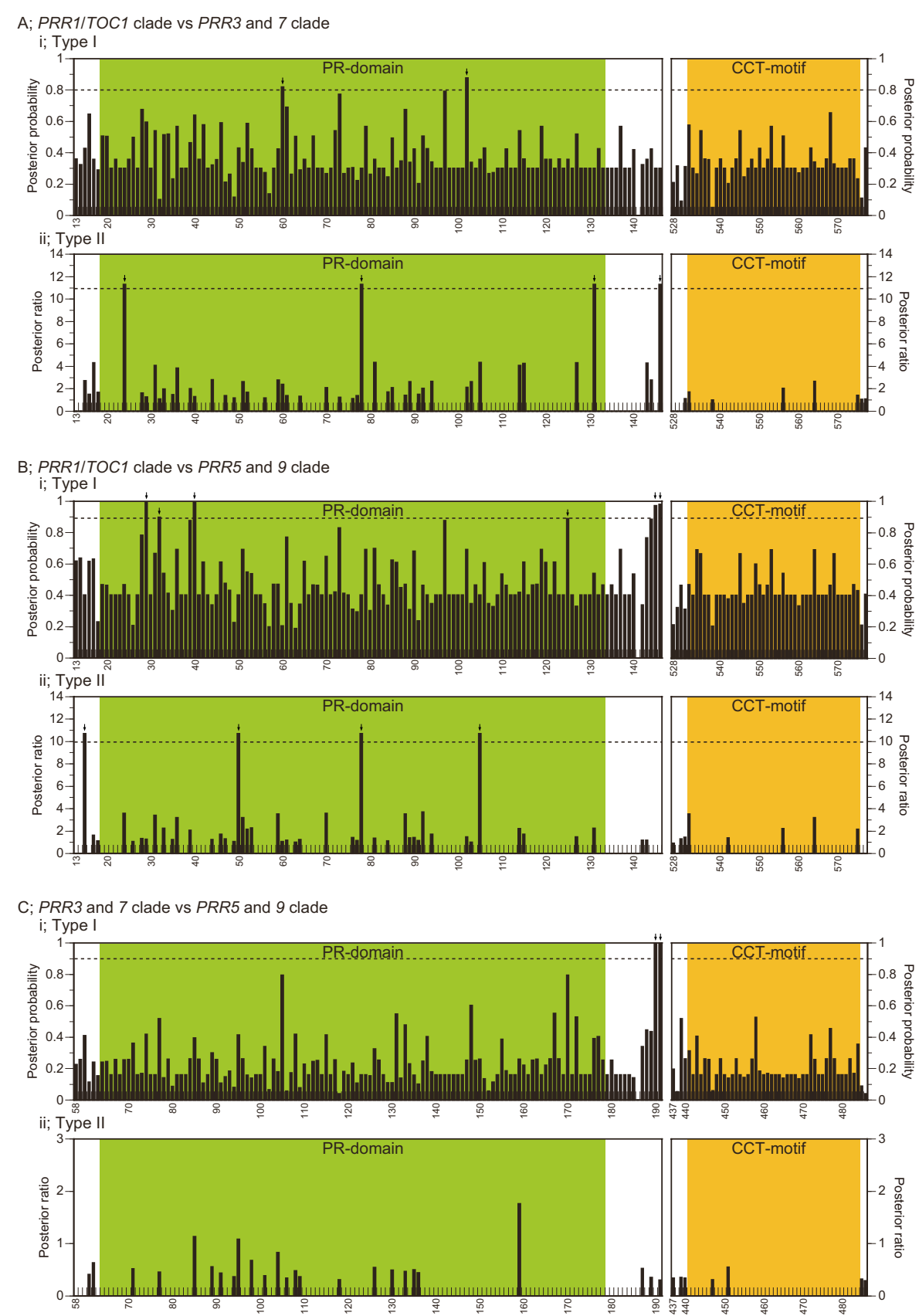

Figure 4 Type I and type II functional divergences among the PRR gene clades. Coefficients of type I (i) and type II (ii) functional divergences between PRR1/TOC1 clade and PRR3 and 7 clade (A), between PRR1/TOC1 clade and PRR5 and 9 clade (B) and between PRR3 and 7 clade and PRR5 and 9 clade (C) were calculated by DIVERGE $2.0[55,56]$ using the TCoffee alignment (see Additional file 3 ) and the ME tree (Figure 2). Right and left panels indicate the PR-domain and its flanking region, and the CCT-motif and its flanking region, respectively. Positions of amino acid residues correspond to AtPRR1/TOC1 in PRR1/TOC1 clade vs PRR3 and 7 clade, AtPRR1/TOC1 in PRR1/TOC1 clade vs PRR5 and 9 clade and to AtPRR3 in PRR3 and 7 clade vs PRR5 and 9 clade. Cutoff values of the posterior probability and posterior ratio were established empirically by sequentially removing the highest scoring sites from the alignment until $\theta=0$. The cutoff values are shown by broken lines. The value of $\theta_{\|}$between $P R R 3$ and 7 clade and $P R R 5$ and 9 clade was not set because the coefficient of the $\theta_{\|}$was not significantly greater than zero. Thus, there is no broken line shown in the bottom panel (C, ii). The regions represented in this figure are surrounded with blue boxes in Additional file 3. Arrows indicate sites above the empirical cutoff values. Green and yellow shadings on the panels indicate PR-domain and CCT-motif, respectively. 


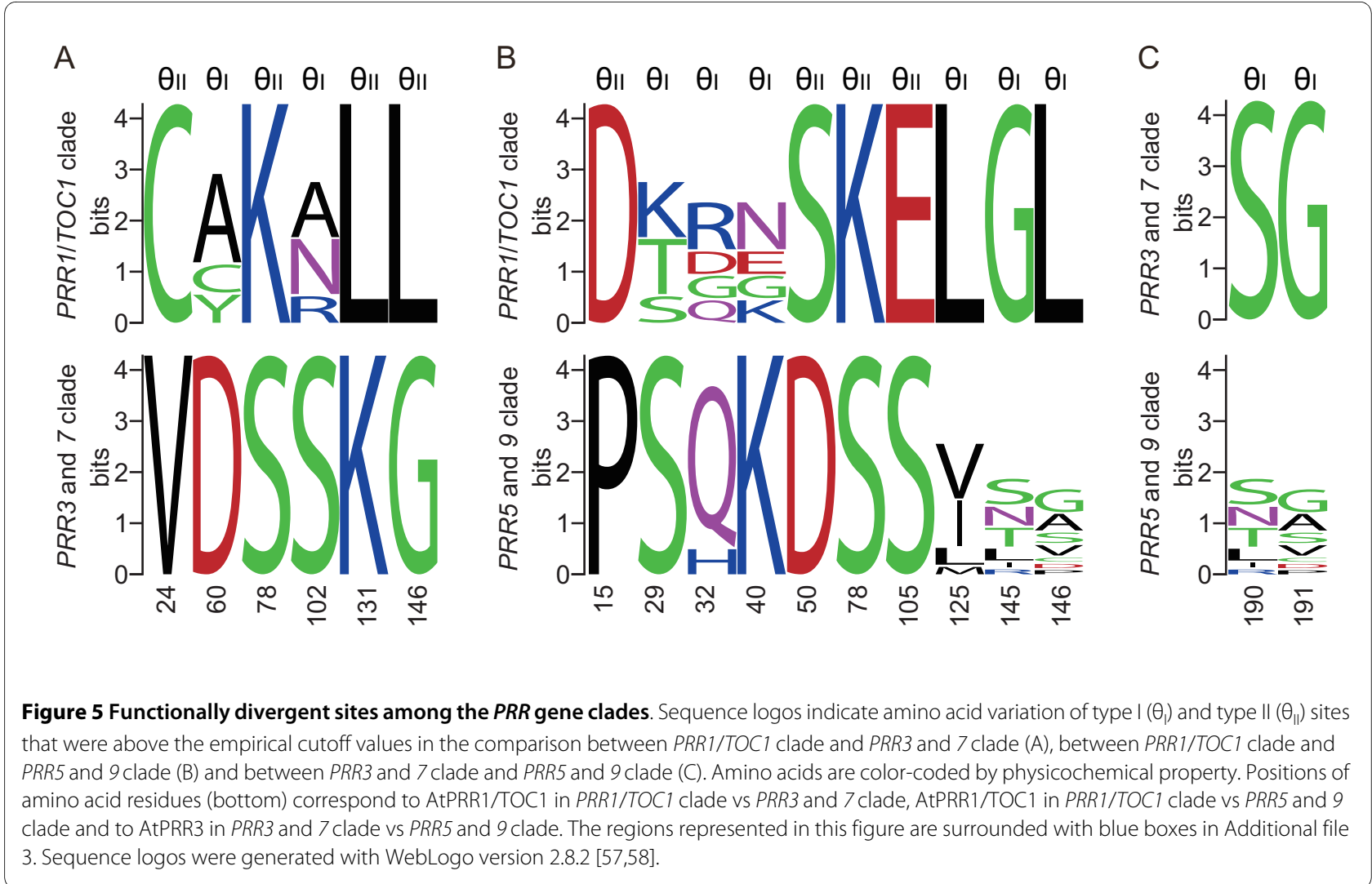

some 8 have lost $P R R$ genes ([18], see also the comparative genomic tool, VISTA Browser [31]). These results indicated that the gene duplication event resulting in PRR37 and PRR73 was the monocotyledonous $\rho$ polyploidy event, and that PRR59 and PRR95 were duplicated via the $\rho$ polyploidy event but one of the duplicated genes was lost from genomes of O. sativa and S. bicolor (Figure 7).

\section{Discussion}

The plant clock system consists of multiple interlocked feedback loops, which are comprised predominantly of two gene families, $L H Y / C C A 1 \mathrm{~s}$ and PRRs [4,5]. These gene families are conserved among both monocots and eudicots [8]. To clarify the evolutionary process of the plant clock system, we recently reported the molecular phylogeny of $L H Y / C C A 1$ genes in angiosperms [9]. Furthermore, in the present study, we reconstructed phylogenetic relationships among clock-associated $P R R$ genes in monocots and eudicots using two approaches: reconstruction of phylogenetic trees and examination of syntenic relationships. Together, these phylogenetic analyses of the plant circadian clock related-genes, $L H Y / C C A 1 \mathrm{~s}$ and $P R R$ s, are promising tools to unravel the evolutionary history of the plant clock system among angiosperm lineages.

\section{Evolutionary processes of clock-associated PRR genes in angiosperms}

$P R R$ genes are conserved in angiosperms and at least five copies of $P R R$ genes have been retained in their genomes (see Additional file 1). Angiosperm $P R R$ genes are grouped into three clades (the PRR1/TOC1 clade, the $P R R 3$ and 7 clade, and the PRR5 and 9 clade) that have already existed prior to the divergence of monocots and eudicots (Figures 2 and 3). After the speciation of monocots and eudicots, copy numbers of PRR genes independently increased in each lineage as a result of ancient chromosomal duplication events (Figure 7). In monocots, the ancestral PRR37/PRR73 was duplicated into PRR37 and $P R R 73$ in the $\rho$ polyploidy event that occurred before the speciation of $O$. sativa and $S$. bicolor $[18,19]$. In eudicots, the gene duplication events between $P R R 3$ and PRR7 and between PRR5 and PRR9/9l are derived from the $\gamma$ polyploidy event that took place before the speciation of Vitales ( $V$. vinifera) and eurosid species ( $P$. trichocarpa, C. papaya, and A. thaliana) [16]. In addition, our results show that PRR genes in P. trichocarpa have expanded more than those in other plant species (see Additional file 1). This expansion apparently resulted from the salicoid polyploidy event that occurred in the Populus lineage but not in other eudicots ( $V$. vinifera, $C$. papaya, and A. thaliana) (Figure 7). Consequently, circa- 
A

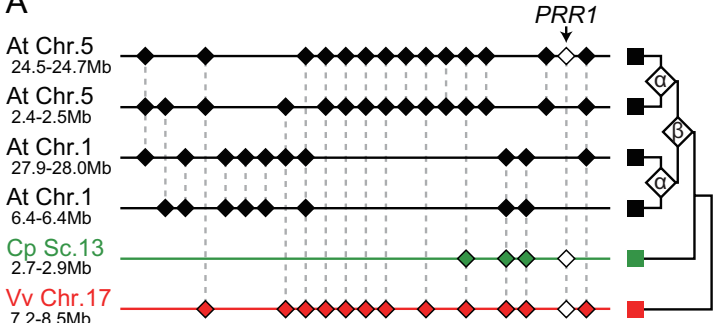

C

At Chr. $24.0-24.5 \mathrm{Mb}$

24.0-24.5M

16.5-17.2Mb

At Chr.1

2.3-2 $5 \mathrm{Mb}$

At Chr.2

12.0-12.5Mb

Cp Sc.139

$0.07-0.31 \mathrm{Mb}$

Pt Chr.1

Pt Chr 9

Pt Chr.9
$8.2-11.3 \mathrm{Mb}$

Vv Chr 17

v $\mathrm{Chr}$.1

D

At Chr. 5
$0.27-0.87 \mathrm{Mb}$

$0.27-0.87 \mathrm{M}$

At Chr.

At Chr.2

16.1-16.4M

At $\mathrm{Chr} .3$

20.1-20.4M

Cp Sc. 1

3.8-4.9M

Pt Chr.8

Pt Chr.10

Vv Chr.13

1.8-6.8Mb

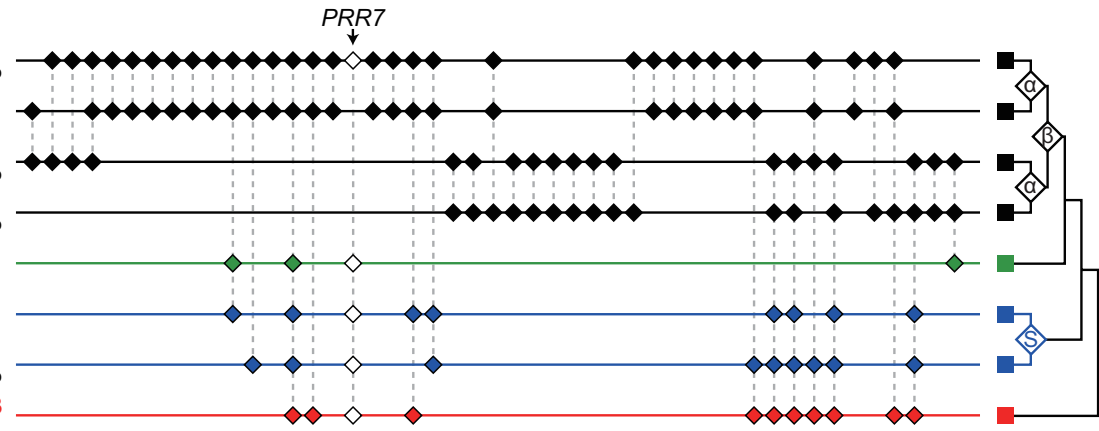

E

At Chr.2

19.0-19.3M

At Chr. 3

22.8-23.0Mb

At Chr. 1

At Chr. 4

$0.35-0.57 \mathrm{M}$

Pt Chr.2

12.4-14.3Mb

Pt Chr.14

Vv Chr.15
7.1-7.6Mb
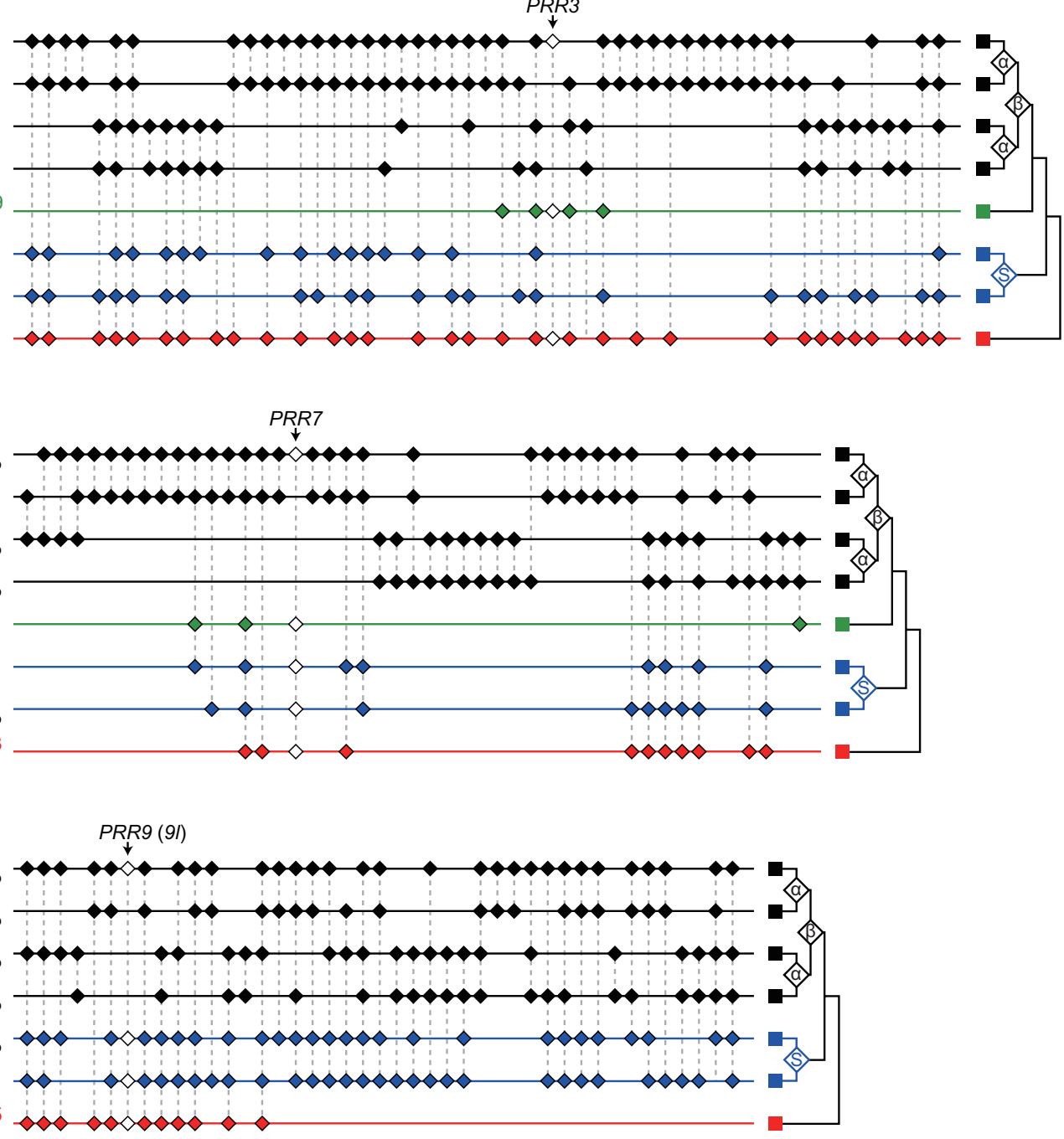

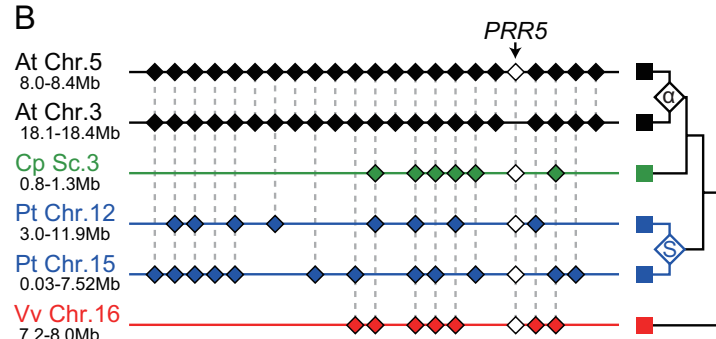

Vv Chr. 16

$\infty \infty \infty$

Figure 6 Chromosomal syntenies of flanking regions of eudicotyledonous $P R R$ genes. Syntenic relationships of flanking regions of $P R R 1 / T O C 1$ (A), PRR5 (B), PRR3 (C), PRR7 (D) and PRR9/9/ (E) were examined using the comparative genomic tool, CoGe [29]. Syntenic relationships within A. thaliana or P. trichocarpa were analyzed by a comparative genomic tool, CoGe, and according to previous studies [17,27,28,59]. Diamonds colored with black (A. thaliana), green (C. papaya), blue (P. trichocarpa) and red ( $V$. vinifera) indicate individual genes. White diamonds marked with arrows indicate PRR genes. Genes with no syntenic matches on the selected regions are not plotted. Orthologous genes are connected by broken lines. Diamonds with characters on the right side of strands indicate angiosperm polyploidy event ( $\alpha, \beta$ and salicoid). The salicoid polyploidy event is shown as the diamond with the initial letter of salicoid (S). The lengths of the genomic regions are shown on the left. 


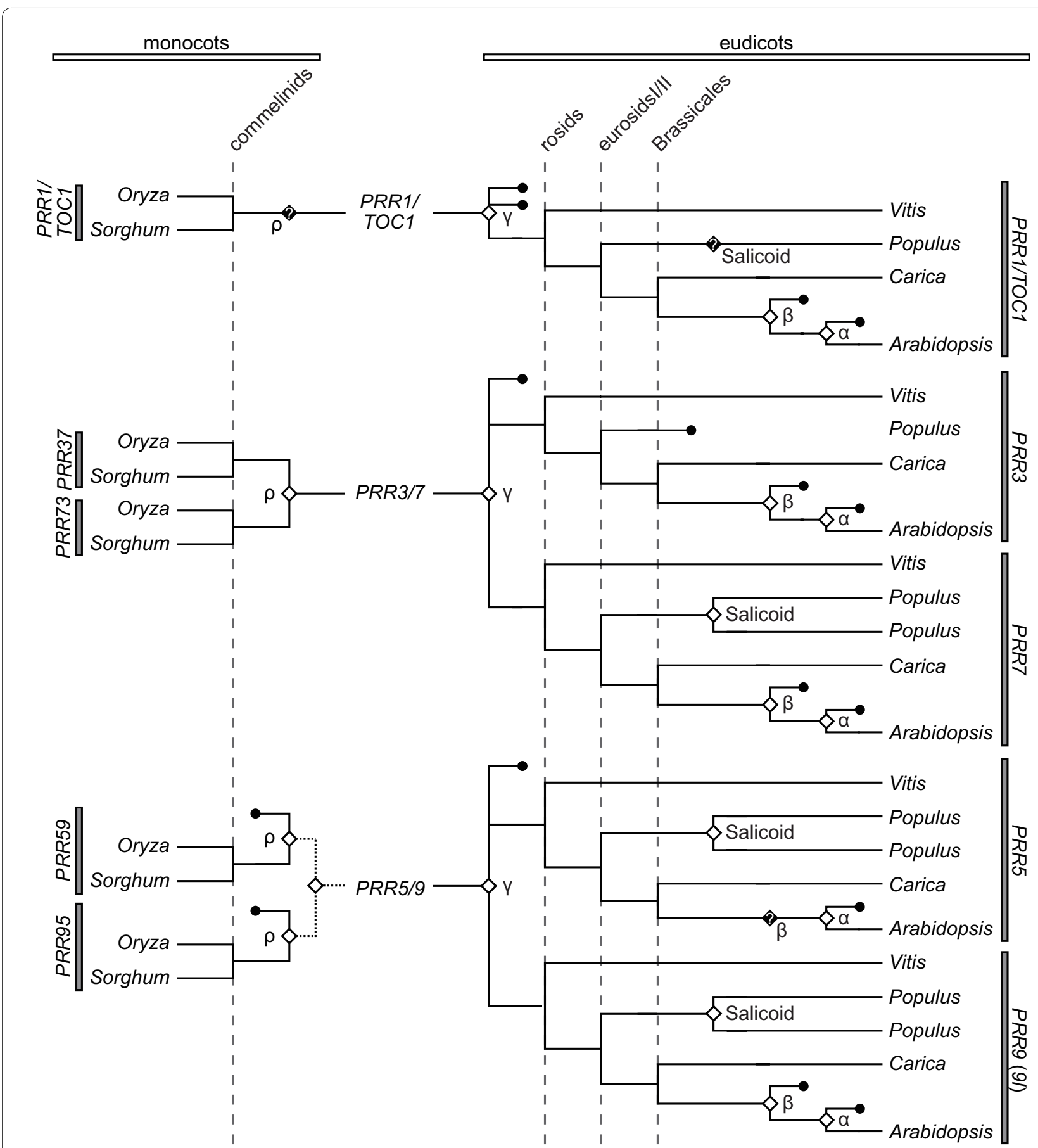

Figure 7 Evolutionary processes of $P R R$ genes in angiosperms reconstructed by phylogenetic analysis and syntenic relationships. Chromosomal syntenies among eudicots or monocots were analyzed by the comparative genomic tool, CoGe [29] or VISTA Browser [31], and according to previous studies $[11,16-18,27,28,30,59]$. Diamonds and circles indicate gene duplication and gene loss event, respectively. The timing of a gene duplication event that is not clear in the previous studies is shown by dotted line. Black diamonds with a question mark indicate that a gene duplication event derived from a polyploidy event is not resolved. Timings of plant speciation in commelinids, rosids, eurosids $1 / \|$ and Brassicales are described by broken lines. 
dian clocks may have become more intricate networks after the speciation of monocots and eudicots if the additional genes have roles in the circadian networks.

In contrast to the increase in $P R R$ genes via ancient chromosomal duplication events, the present genome of A. thaliana retains one copy of each $P R R$ gene (PRR1/ TOC1, PRR3, 5, 7, and 9) after $\beta$ and $\alpha$ polyploidy events (Figure 7). Likewise, in Brassica rapa, PRR genes that were increased in a recent hexaploidization event have been reduced in its genome following the event, though the genome retains at least a set of $P R R$ genes [32]. These results implied that dosages of the clock related-genes had been altered in the genomes during evolution. Since some reports showed that a gene dosage change of clock related genes influenced the clock regulatory network and downstream signals [33,34], plants may have flexibly modulated the complex network of the clock system after polyploidy events and subsequent gene deletion events. Indeed, allopolyploid Arabidopsis species can fine-tune a regulatory and stoichiometric balance of the circadian clock system to properly maintain a downstream homeostasis of the plants [35]. The history of gene duplications and deletions in the Arabidopsis clock system imply that the regulatory network of the clock system has maintained a degree of organization throughout the dynamic changes of copy numbers and functions of clock-related genes.

\section{Phylogenetic footprint of the plant clock system in angiosperms}

Loops I and III of the Arabidopsis clock system contain four PRR genes (PRR1/TOC1, PRR3, 7 and 9) and two $L H Y / C C A 1$ genes (LHY and CCA1) (Figure 1) [4,5,36]. Although the circadian clock-related genes in A. thaliana were duplicated via the $\beta$ and $\alpha$ polyploidy events, the present genome of $A$. thaliana retains only one pair of $L H Y$ and $C C A 1$ genes, which is derived from the $\beta$ polyploidy event (Figure 8) [9]. As the $\beta$ polyploidy event is assumed to have occurred in the Brassicaceae, $L H Y$ and CCA1 genes did not diverge before the speciation of $A$. thaliana and C. papaya, which is consistent with the fact that there is only one copy of the $L H Y / C C A 1$ gene in the genome of $C$. papaya [11]. Similar to the genome of $A$. thaliana, the $C$. papaya genome retained only one copy each of the PRR1/TOC1, PRR3, 7, and 9 genes (see Additional file 1). These results suggest that one copy of $L H Y /$ CCA1, PRR1/TOC1, PRR3, 7, and 9 was involved in the plant clock system in the common ancestor of $A$. thaliana and C. papaya.

The evolutionary history of the plant clock system in two divergent members of Brassicales (A. thaliana and $C$. papaya) raises the question as to when the Arabidopsistype clock apparatus arose in the evolutionary history of plants. Phylogenies of the circadian clock-related genes showed that a set of the genes, one copy of each $L H Y$ / CCA1, PRR1/TOC1, PRR3, 7 and $9 l$ genes, is conserved in the genome of $V$. vinifera (Figures 7 and 8, see Additional file 1) $[9,16]$. Conservation of the set of clockrelated genes suggests that the fundamental mechanism of the Arabidopsis-type clock apparatus was formed before the speciation of Vitales ( $V$. vinifera) and eurosid species ( $P$. trichocarpa, C. papaya, and $A$. thaliana), although it remains to be determined whether functional divergences between PRR3 and 7 and between PRR5 and $9 / 9 l$, which were duplicated in the $\gamma$ triplication event, existed in the common ancestor of Vitales and eurosids (Figure 8).

Intriguingly, the clock system of $P$. trichocarpa might differ from the Arabidopsis clock system, because the $P$. trichocarpa PRR3 gene was lost and LHY/CCA1 and $P R R 7$ and 9 were duplicated via the salicoid polyploidy event that occurred after the speciation of eurosids I and II (Figures 7 and 8). PRR3 protein in $A$. thaliana interacts with PRR1/TOC1 protein, which is a component of the main loop (Loop I), to inhibit its protein degradation [36]. The lack of PRR3 in Populus might influence the posttranslational regulation of PRR1/TOC1 protein or might be compensated by recruiting other $P R R$ genes although conserved changes that would mark the $P R R 3$ gene cluster were not identified in other Populus PRR proteins (data not shown). Furthermore, duplication of $L H Y$ / CCA1 and PRR7 and 9, but not PRR1/TOC1, could also affect the regulation mechanism of the Populus clock system, because a dosage balance in the plant clock system was ruined [33]. We recently revealed that Populus $L H Y \mathrm{~S}$ show typical diurnal expressions similar to $L H Y / C C A 1$ genes in other plant species [9,37-40], which is apparently contradictory to the speculation. The Populus clock system appears to retain a functional regulatory network in regard to the expression regulation of the $L H Y$ genes throughout the evolutionary changes of the circadian clock components.

The monocots $O$. sativa and S. bicolor retain one $L H Y$ / CCA1 gene and five $P R R$ genes in their genomes (see Additional file 1) [9]. Phylogenetic analysis showed that the gene duplication events that produced $P R R 37$ and 73 , and PRR59 and 95 in monocots occurred separately and independently of the events that produced $P R R 3$ and 7 , and PRR5 and 9 in eudicots (Figure 7). This finding raises a complex question; what are the roles of these paralogous genes in the monocotyledonous clock system? The expression of PRR5 in A. thaliana is not regulated by light signals and reaches a peak of the diurnal rhythm around 8 $\mathrm{h}$ after dawn [10]. On the other hand, PRR9 in A. thaliana shows a light response expression, resulting in rhythmicity with peak expression just after dawn [10,41]. PRR59 and 95 in $O$. sativa have peak expressions around $9 \mathrm{~h}$ after dawn and are not induced by light signals [13], which 


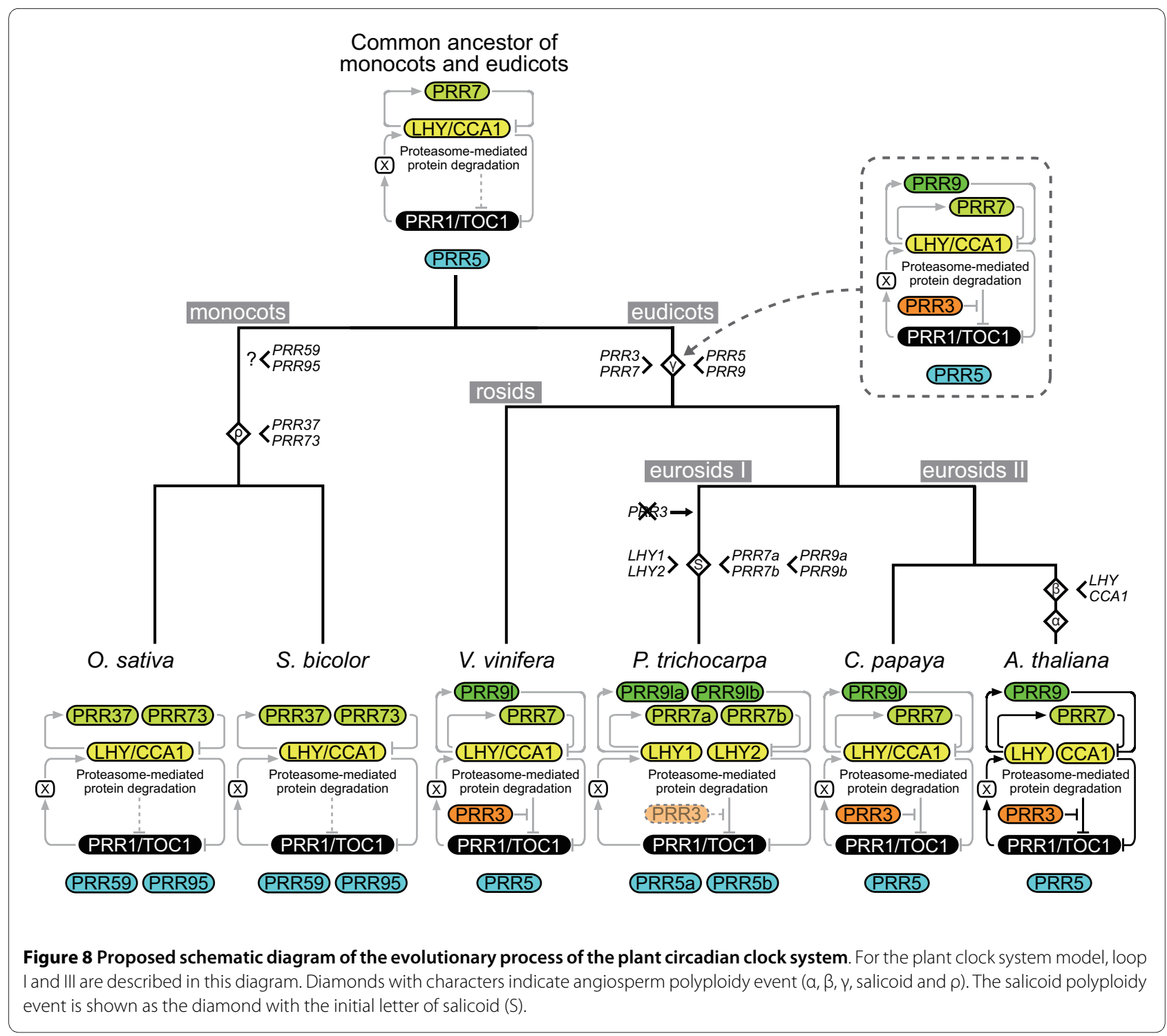

appear to be more similar to the regulation of $A$. thaliana PRR5 expression than to that of $A$. thaliana PRR9 expression. In addition, peak expressions of $O$. sativa PRR37 and 73 are followed by expressions of PRP59 and 95, which may correspond to the sequential expression pattern of $A$. thaliana PRR7 and PRR5 [10,13]. These results collectively suggest that paralogous gene pairs $P R R 59 / 95$ and $P R R 37 / 73$ genes in monocots share functional roles with PRR5 and PRR7, respectively, in A. thaliana (Figure 8). Together, these data indicate that a common ancestor of monocots and eudicots may have had a main feedback loop (LHY/CCA1-PRR1/TOC1) that was not posttranslationally regulated by $P R R 3$. Although the ancestral clock system appears to have been more simplified than that of the current Arabidopsis-type clock apparatus, it is assumed that the ancestral clock system have had the basic components reconstructing a primitive multiple feedback loop system.

\section{Conclusions}

The present study inferred the molecular phylogeny of angiosperm $P R R$ genes that have key roles in the plant clock system. Clock-associated PRR genes diverged into three clades before the speciation of monocots and eudicots and, in addition, $P R R 3 / 7$ and $P R R 5 / 9$ underwent independent expansion in monocots and eudicots (Figure 7). Taken together with the molecular phylogeny of $L H Y$ / CCA1 genes [9], our studies suggest that the basic components of the Arabidopsis clock were established prior to the speciation of eudicots and monocots (Figure 8). Additional functional analyses and accumulation of genomic information from other plant species will clarify 
details of evolutionary and developmental processes of plant clock systems.

\section{Methods}

Retrieval of sequences of clock-associated PRR genes from draft genome sequences

$P R R$ genes were retrieved from genomic databases for $A$. thaliana (TIGR Arabidopsis thaliana Database [42]) and O. sativa (Rice Annotation Project Database [43]). To identify PRR genes in S. bicolor, V. vinifera, P. trichocarpa, and $C$. papaya, TBLASTN searches were performed against the genomic databases using amino acid sequences encoded by $P R R$ genes of $A$. thaliana or $O$. sativa as queries: JGI Sorghum bicolor v1.0 [44] for $S$. bicolor; Grape Genome Browser [45] for $V$. vinifera; JGI Populus trichocarpa v1.1 [46] for P. trichocarpa, and Papaya Genome Project v0.4 in CoGe [29] for C. papaya. Genes that retained the typical PR-domain at the N-terminal region and the CCT-motif at the $\mathrm{C}$-terminal region were retrieved from these genomic databases. Genes that lacked the PR-domain or CCT-motif but showed significantly high similarity with typical $P R R$ genes were also retrieved (those with E-values lower than $10^{-50}$ or $>90 \%$ similarity). The genes retrieved from the genomic databases were aligned with $P R R$ genes in A. thaliana and $O$. sativa using the TCoffee program [47]. Mispredicted genes, if found, were manually modified as follows. For predicted genes lacking a conserved portion of the $P R R$ gene, we searched the database for expressed sequence tags (ESTs) of the gene (TIGR Plant Transcript Assemblies [48]) and re-annotated by assembling the predicted gene and relevant ESTs. In some cases, the open reading frame of the gene was repredicted by the Fgenesh+ program [49]. When the exon-intron boundary of a gene was mis-demarcated, we improved the boundary based on standard donor/acceptor splice sites without resulting in a frame shift.

\section{Phylogenetic analysis}

Amino acid sequences were deduced from nucleotide sequences of the predicted $P R R$ genes and then aligned using the TCoffee program [47]. The alignment around the flanking region of the PR-domain was manually corrected based on the exon-intron structure. The number of amino acids substituted between each pair of PRR proteins was estimated by the Jones-Taylor-Thornton (JTT) model [50] with the complete- deletion option. From the number of estimated amino acid substitutions, a phylogenetic tree was reconstructed by the ME method [51]. Bootstrap values were calculated with 1,000 replications using the ME method [52]. PRR1/TOC1 genes were utilized as an outgroup in the phylogenetic trees. These procedures were performed using the MEGA4 software $[53,54]$.

\section{Detection of functional divergence}

Type I and type II functional divergences among $P R R$ gene clades was examined using the DIVERGE 2.0 software $[24,55,56]$. To calculate coefficient of type I and type II functional divergences $\left(\theta_{\mathrm{I}}\right.$ and $\left.\theta_{\text {II }}\right)$, we used the protein sequence alignment constructed using the TCoffee program [47] and the topology and branch length of phylogenetic tree reconstructed by the ME method. Cutoff values of the posterior probability and posterior ratio were set empirically by sequentially removing the highest scoring sites from the alignment until $\theta=0$. Sequence logos were generated with WebLogo version 2.8.2 [57,58].

\section{Identification of chromosomal synteny}

Conservation of chromosomal synteny in $V$. vinifera, $P$. trichocarpa, C. papaya, and A. thaliana was determined as follows. First, we reconstructed the ancient gene organization of the flanking regions of $A$. thaliana PRR genes before the $\alpha$ and $\beta$ polyploidy events using the chromosomal syntenies reported in previous studies $[27,28]$. Then, we compared the syntenic relationships between the ancient gene organization in A. thaliana and the flanking regions of $P R R$ genes in $V$. vinifera, $P$. trichocarpa, and $C$. papaya using the comparative genomic tool, CoGe $[11,29,59]$. This process also reconstructed chromosomal syntenies in $P$. trichocarpa that were derived from the salicoid polyploidy event [17]. To clarify syntenic relationships derived from the $\gamma$ polyploidy event, we used information on chromosomal syntenies within the genome of $V$. vinifera [16] and the comparative genomic tool, CoGe [29].

Syntenic relationships between the flanking regions of $P R R$ genes in O. sativa and those in S. bicolor were reconfirmed using the chromosomal syntenies reported in previous studies [30] and a comparative genomic tool VISTA Browser [31]. To reconstruct chromosomal syntenies of the flanking regions of $P R R$ genes derived from the monocotyledonous $\rho$ polyploidy event, syntenic regions were identified according to methods reported previously using the $O$. sativa genomic sequence [18].

\section{Additional material}

\footnotetext{
Additional file 1 PRR genes in angiosperms used in the present study. aPlant classification refers to APGII [61]. bGene ID corresponds to the name obtained from Rice Annotation Project Database [43]. 'Gene ID corresponds to the name obtained from JGI Sorghum bicolor v1.0 [44]. ' $G$ Gene IDs correspond to the names obtained from TIGR Plant Transcript Assemblies [48]. eGene ID corresponds to the name obtained from Grape Genome Browser [45]. ${ }^{\mathrm{f}}$ Gene IDs correspond to the names obtained from JGI Populus trichocarpa v1.1 [46]. 9Gene IDs correspond to the names obtained from TIGR Plant Genomics [62]. hGene IDs correspond to the names obtained from Comparative Genomics Homepage [29]. ${ }^{*}$ Genes, which appeared to be misprediced, were manually modified in this study. ${ }^{* *}$ Gene is misannotated. ${ }^{* *}$ Genes lack the PR-domain.
} 


\begin{abstract}
Additional file $\mathbf{2}$ Predicted $P R R$ genes in angiosperms. Angiosperm $P R R$ genes were retrieved from genomic databases for $A$. thaliana (TIGR Arabidopsis thaliana Database, http://www.tigr.org/tdb/e2k1/ath1/), V. vinifera (Grape Genome Browser, http://www.genoscope.cns.fr/externe/GenomeBrowser/Vitis/), P. trichocarpa (JGl Populus trichocarpa v1.1, http:// genome.jgi-psf.org/Poptr1 1/Poptr1 1.home.html), C. papaya (Papaya Genome Project v0.4 in CoGe, http://synteny.cnr.berkeley.edu/CoGe/, O. sativa (Rice Annotation Project Database, http://rapdb.dna.affrc.go.jp/) and S. bicolor (JGI Sorghum bicolor v1.0, http://genome.jgi-psf.org/Sorbi1/ Sorbi1.home.html).

Additional file 3 Alignments of the amino acid sequences encoded by angiosperm PRR genes. Amino acid sequences were aligned using TCoffee program http://www.ebi.ac.uk/t-coffee/. Amino acid conservation was highlighted by the boxshade program http://www.ch.embnet.org/software/BOX form.html. Identical and similar amino acid residues are highlighted with black and gray shading, respectively. Blue boxes indicate the regions that were represented in Figures 4 and 5 .
\end{abstract}

Additional file 4 Alignment of the amino acid sequences encoded by (A)AtPRR9s and (B)PtPRR5s. Sequence similarity is indicated below the alignment using the symbols "asterisk," "colon," and "dot" for identical, highly similar, and weakly similar residues, respectively. Black shadings indicate the PR-domain and the CCT-motif. Accession numbers and gene IDs of the PRR genes are shown in Additional file 1.

\section{Additional file 5 Nucleotide sequences around the region of the exon-intron boundaries of angiosperm PRR genes at the flanking region of PR-domain. Black and gray shadings on the alignments indicate a site of exon-intron boundary and one-amino acid deletion, respectively. Higher degree of conservation of nucleotide sequence is shown by the big ger size of letters}

Additional file 6 Phylogenetic tree of $P R R$ genes reconstructed by the Neighbor-Joining (NJ) method. Full-length amino acid sequences were aligned using TCoffee program. The phylogenetic tree was reconstructed by the NJ method from the numbers of amino acid substitutions estimated by applying the JTT model. The numerals at the branch indicate bootstrap values calculated by the $\mathrm{NJ}$ method with 1,000 replications. Bootstrap values $>50 \%$ are shown.

Additional file 7 Phylogenetic trees of $P R R$ genes reconstructed by the Maximum likelihood (ML) and Bayesian methods. Full-length amino acid sequences were aligned using TCoffee program. The phylogenetic trees were reconstructed by the ML and Bayesian methods with applying the JTT model. The ML and Bayesian analyses were performed using the PhyML http://www.atgc-montpellier.fr/ and MrBayes http:// mrbayes.csit.fsu.edu/ programs, respectively. PRR1/TOC1 genes were utilized as an outgroup in the phylogenetic trees. Support for the branches was calculated as percent of 100 bootstrap replications of the ML method (left) and Baysian posterior probabilities (right). The values $>50 \%$ are shown.

\section{Authors' contributions}

NT conceived of the study, performed all analyses and wrote the manuscript. MU, SS and CTS participated in coordination and helped to draft the manuscript. All authors read and approved the final manuscript.

\section{Acknowledgements}

We thank Dr. Maria E. Eriksson (Umeå Plant Science Centre, Umeå University) for critical reading of the manuscript. This work was supported in part by a Grant-in-Aid for the 21st Century COE Program from the Ministry of Education, Culture, Sports, Science and Technology of Japan (K-3 to MU) and by a grant from the Japan Society for the Promotion of Science (19.9498 to NT).

\section{Author Details}

'United Graduate School of Agricultural Sciences, Iwate University, Morioka 020-8550, Japan, 2Laboratory of Bioscience, Faculty of Engineering, Iwate University, Morioka 020-8551, Japan, ${ }^{3}$ Cryobiofrontier Research Center, Iwate University, Morioka 020-8550, Japan, 4 Umeå Plant Science Centre, Department of Plant Physiology, Umeå University, SE-901 87 Umeå, Sweden and ${ }^{5}$ Department of Bioenvironmental Science, Okazaki Institute for Integrative Bioscience, National Institutes of Natural Sciences, Okazaki 444-8787, Japan

Received: 30 June 2009 Accepted: 1 May 2010

Published: 1 May 2010

\section{References}

1. Yakir E, Hilman D, Harir Y, Green RM: Regulation of output from the plant circadian clock. FEBS J 2007, 274:335-345.

2. McClung CR: Plant circadian rhythms. Plant Cell 2006, 18:792-803.

3. Más P: Circadian clock signaling in Arabidopsis thaliana: from gene expression to physiology and development. Int J Dev Biol 2005, 49:491-500.

4. Locke JC, Kozma-Bognár L, Gould PD, Fehér B, Kevei E, Nagy F, Turner MS, Hall A, Millar AJ: Experimental validation of a predicted feedback loop in the multi-oscillator clock of Arabidopsis thaliana. Mol Syst Biol 2006, 2:59.

5. Zeilinger MN, Farré EM, Taylor SR, Kay SA, Doyle FJ: A novel computational model of the circadian clock in Arabidopsis that incorporates PRR7 and PRR9. Mol Syst Biol 2006, 2:58.

6. Alabadí D, Oyama T, Yanovsky MJ, Harmon FG, Más P, Kay SA: Reciprocal regulation between $T O C 1$ and $L H Y / C C A 1$ within the Arabidopsis circadian clock. Science 2001, 293:880-883.

7. McClung CR: Comes a time. Curr Opin Plant Biol 2008, 11:514-520.

8. Murakami M, Tago Y, Yamashino T, Mizuno T: Comparative overviews of clock-associated genes of Arabidopsis thaliana and Oryza sativa. Plant Cell Physiol 2007, 48:110-121.

9. Takata N, Saito S, Saito CT, Nanjo T, Shinohara K, Uemura M: Molecular phylogeny and expression of poplar circadian clock genes, LHY1 and LHY2. New Phytol 2009, 181:808-819.

10. Matsushika A, Makino S, Kojima M, Mizuno T: Circadian waves of expression of the APRR1/TOC 1 family of pseudo-response regulators in Arabidopsis thaliana: insight into the plant circadian clock. Plant Cell Physiol 2000, 41:1002-1012.

11. Ming R, Hou S, Feng Y, Yu Q, Dionne-Laporte A, Saw JH, Senin P, Wang W, Ly BV, Lewis KL, Salzberg SL, Feng L, Jones MR, Skelton RL, Murray JE, Chen C, Qian W, Shen J, Du P, Eustice M, Tong E, Tang H, Lyons E, Paull RE, Michael TP, Wall K, Rice DW, Albert H, Wang ML, Zhu YJ, Schatz M, Nagarajan N, Acob RA, Guan P, Blas A, Wai CM, Ackerman CM, Ren Y, Liu C, Wang J, Wang J, Na JK, Shakirov EV, Haas B, Thimmapuram J, Nelson D, Wang X, Bowers JE, Gschwend AR, Delcher AL, Singh R, Suzuki JY, Tripathi S, Neupane K, Wei H, Irikura B, Paidi M, Jiang N, Zhang W, Presting G, Windsor A, Navajas-Pérez R, Torres MJ, Feltus FA, Porter B, Li Y, Burroughs AM, Luo MC, Liu L, Christopher DA, Mount SM, Moore PH, Sugimura T, Jiang J, Schuler MA, Friedman V, Mitchell-Olds T, Shippen DE, dePamphilis CW, Palmer JD, Freeling M, Paterson AH, Gonsalves D, Wang L, Alam M: The draft genome of the transgenic tropical fruit tree papaya (Carica papaya Linnaeus). Nature 2008, 452:991-996.

12. Ramírez-Carvajal GA, Morse AM, Davis JM: Transcript profiles of the cytokinin response regulator gene family in Populus imply diverse roles in plant development. New Phytol 2008, 177:77-89.

13. Murakami M, Ashikari M, Miura K, Yamashino T, Mizuno T: The evolutionarily conserved OSPRR quintet: rice pseudo-response regulators implicated in circadian rhythm. Plant Cell Physiol 2003, 44:1229-1236.

14. Tang H, Bowers JE, Wang X, Ming R, Alam M, Paterson AH: Synteny and collinearity in plant genomes. Science 2008, 320:486-488.

15. Arabidopsis Genome Initiative: Analysis of the genome sequence of the flowering plant Arabidopsis thaliana. Nature 2000, 408:796-815.

16. Jaillon O, Aury JM, Noel B, Policriti A, Clepet C, Casagrande A, Choisne N, Aubourg S, Vitulo N, Jubin C, Vezzi A, Legeai F, Hugueney P, Dasilva C, Horner D, Mica E, Jublot D, Poulain J, Bruyère C, Billault A, Segurens B, Gouyvenoux M, Ugarte E, Cattonaro F, Anthouard V, Vico V, Del Fabbro C, Alaux M, Di Gaspero G, Dumas V, Felice N, Paillard S, Juman I, Moroldo M, Scalabrin S, Canaguier A, Le Clainche I, Malacrida G, Durand E, Pesole G, Laucou V, Chatelet P, Merdinoglu D, Delledonne M, Pezzotti M, Lecharny A, Scarpelli C, Artiguenave F, Pè ME, Valle G, Morgante M, Caboche M, Adam-Blondon AF, Weissenbach J, Quétier F, Wincker P, French-Italian Public Consortium for Grapevine Genome Characterization: The grapevine genome sequence suggests ancestral hexaploidization in major angiosperm phyla. Nature 2007, 449:463-467.

17. Tuskan GA, Difazio S, Jansson S, Bohlmann J, Grigoriev I, Hellsten U, Putnam N, Ralph S, Rombauts S, Salamov A, Schein J, Sterck L, Aerts A, Bhalerao RR, Bhalerao RP, Blaudez D, Boerjan W, Brun A, Brunner A, Busov V, Campbell M, Carlson J, Chalot M, Chapman J, Chen GL, Cooper D, Coutinho PM, Couturier J, Covert S, Cronk Q, Cunningham R, Davis J, Degroeve S, Déjardin A, Depamphilis C, Detter J, Dirks B, Dubchak I, Duplessis S, Ehlting J, Ellis B, Gendler K, Goodstein D, Gribskov M, Grimwood J, Groover A, Gunter L, Hamberger B, Heinze B, Helariutta Y, 
Henrissat B, Holligan D, Holt R, Huang W, Islam-Faridi N, Jones S, JonesRhoades M, Jorgensen R, Joshi C, Kangasjärvi J, Karlsson J, Kelleher C, Kirkpatrick R, Kirst M, Kohler A, Kalluri U, Larimer F, Leebens-Mack J, Leplé JC, Locascio P, Lou Y, Lucas S, Martin F, Montanini B, Napoli C, Nelson DR, Nelson C, Nieminen K, Nilsson O, Pereda V, Peter G, Philippe R, Pilate G, Poliakov A, Razumovskaya J, Richardson P, Rinaldi C, Ritland K, Rouzé P, Ryaboy D, Schmutz J, Schrader J, Segerman B, Shin H, Siddiqui A, Sterky F, Terry A, Tsai CJ, Uberbacher E, Unneberg P, Vahala J, Wall K, Wessler S, Yang G, Yin T, Douglas C, Marra M, Sandberg G, Peer Y Van de, Rokhsar D: The genome of black cottonwood, Populus trichocarpa (Torr. \& Gray). Science 2006, 313:1596-1604.

18. Salse J, Bolot S, Throude M, Jouffe V, Piegu B, Quraishi UM, Calcagno T, Cooke R, Delseny M, Feuillet C: Identification and characterization of shared duplications between rice and wheat provide new insight into grass genome evolution. Plant Cell 2008, 20:11-24.

19. Yu J, Wang J, Lin W, Li S, Li H, Zhou J, Ni P, Dong W, Hu S, Zeng C, Zhang J, Zhang Y, Li R, Xu Z, Li S, Li X, Zheng H, Cong L, Lin L, Yin J, Geng J, Li G, Shi J, Liu J, Lv H, Li J, Wang J, Deng Y, Ran L, Shi X, Wang X, Wu Q, Li C, Ren X, Wang J, Wang X, Li D, Liu D, Zhang X, Ji Z, Zhao W, Sun Y, Zhang Z, Bao J, Han Y, Dong L, Ji J, Chen P, Wu S, Liu J, Xiao Y, Bu D, Tan J, Yang L, Ye C, Zhang J, Xu J, Zhou Y, Yu Y, Zhang B, Zhuang S, Wei H, Liu B, Lei M, Yu H, Li Y, Xu H, Wei S, He X, Fang L, Zhang Z, Zhang Y, Huang X, Su Z, Tong W, Li J, Tong Z, Li S, Ye J, Wang L, Fang L, Lei T, Chen C, Chen H, Xu Z, Li H, Huang H, Zhang F, Xu H, Li N, Zhao C, Li S, Dong L, Huang Y, Li L, Xi Y, Qi Q, Li W, Zhang B, Hu W, Zhang Y, Tian X, Jiao Y, Liang X, Jin J, Gao L, Zheng W, Hao B, Liu S, Wang W, Yuan L, Cao M, McDermott J, Samudrala R, Wang J, Wong $\mathrm{GK}$, Yang H: The Genomes of Oryza sativa: a history of duplications. PLOS Biol 2005, 3:266-281.

20. Adams KL, Wendel JF: Polyploidy and genome evolution in plants. Curr Opin Plant Biol 2005, 8:135-141.

21. Bocock PN, Morse AM, Dervinis C, Davis JM: Evolution and diversity of invertase genes in Populus trichocarpa. Planta 2008, 227:565-576.

22. Sampedro J, Lee Y, Carey RE, dePamphilis C, Cosgrove DJ: Use of genomic history to improve phylogeny and understanding of births and deaths in a gene family. Plant $J 2005,44: 409-419$.

23. Gu X: Maximum-likelihood approach for gene family evolution under functional divergence. Mol Biol Evol 2001, 18:453-464.

24. Gu X: A simple statistical method for estimating type-II (clusterspecific) functional divergence of protein sequences. Mol Biol Evol 2006, 23:1937-1945

25. Ito S, Matsushika A, Yamada H, Sato S, Kato T, Tabata S, Yamashino T, Mizuno T: Characterization of the APRR9 pseudo-response regulator belonging to the APRR1/TOC1 quintet in Arabidopsis thaliana. Plant Cell Physiol 2003, 44:1237-1245.

26. Kiba T, Henriques R, Sakakibara H, Chua NH: Targeted degradation of PSEUDO-RESPONSE REGULATOR5 by an SCFZTL complex regulates clock function and photomorphogenesis in Arabidopsis thaliana. Plant Cell 2007, 19:2516-2530.

27. Bowers JE, Chapman BA, Rong J, Paterson AH: Unravelling angiosperm genome evolution by phylogenetic analysis of chromosomal duplication events. Nature 2003, 422:433-438

28. Thomas BC, Pederson B, Freeling M: Following tetraploidy in an Arabidopsis ancestor, gene were removed preferentially from one homeolog leaving clusters enriched in dose-sensitive genes. Genome Res 2006, 16:934-946.

29. CoGe [http://synteny.cnr.berkeley.edu/CoGe/]

30. Paterson AH, Bowers JE, Bruggmann R, Dubchak I, Grimwood J, Gundlach H, Haberer G, Hellsten U, Mitros T, Poliakov A, Schmutz J, Spannagl M, Tang H, Wang X, Wicker T, Bharti AK, Chapman J, Feltus FA, Gowik U, Grigoriev IV, Lyons E, Maher CA, Martis M, Narechania A, Otillar RP, Penning BW, Salamov AA, Wang Y, Zhang L, Carpita NC, Freeling M, Gingle AR, Hash CT, Keller B, Klein P, Kresovich S, McCann MC, Ming R, Peterson DG, Mehboob-ur-Rahman, Ware D, Westhoff P, Mayer KF, Messing J, Rokhsar DS: The Sorghum bicolor genome and the diversification of grasses. Nature 2009, 457:551-556.

31. VISTA Browser [http://genome.lbl.gov/vista/index.shtml]

32. Kim JA, Yang TJ, Kim JS, Park JY, Kwon SJ, Lim MH, Jin M, Lee SC, Lee SI, Choi BS, Um SH, Kim HI, Chun C, Park BS: Isolation of circadian-associated genes in Brassica rapa by comparative genomics with Arabidopsis thaliana. Mol Cells 2007, 23:145-153.
33. Más P, Alabadí D, Yanovsky MJ, Oyama T, Kay SA: Dual role of TOC1 in the control of circadian and photomorphogenic responses in Arabidopsis. Plant Cell 2003, 15:223-236.

34. Somers DE, Kim WY, Geng R: The F-box protein ZEITLUPE confers dosage-dependent control on the circadian clock, photomorphogenesis, and flowering time. Plant Cell 2004, 16:769-782.

35. Ni Z, Kim ED, Ha M, Lackey E, Liu J, Zhang Y, Sun Q, Chen ZJ: Altered circadian rhythms regulate growth vigour in hybrids and allopolyploids. Nature 2009, 457:327-331.

36. Fujiwara S, Wang L, Han L, Suh SS, Salomé PA, McClung CR, Somers DE: Post-translational regulation of the Arabidopsis circadian clock through selective proteolysis and phosphorylation of pseudo-response regulator proteins. J Biol Chem 2008, 283:23073-23083.

37. Izawa T, Oikawa T, Sugiyama N, Tanisaka T, Yano M, Shimamoto K: Phytochrome mediates the external light signal to repress $F T$ orthologs in photoperiodic flowering of rice. Genes Dev 2002, 16:2006-2020.

38. Kaldis AD, Kousidis P, Kesanopoulos K, Prombona A: Light and circadian regulation in the expression of $L H Y$ and $L h c b$ genes in Phaseolus vulgaris. Plant Mol Biol 2003, 52:981-997.

39. Ramos A, Pérez-Solís E, Ibáñez C, Casado R, Collada C, Gómez L, Aragoncillo C, Allona I: Winter disruption of the circadian clock in chestnut. Proc Natl Acad Sci USA 2005, 102:7037-7042.

40. Wang ZY, Tobin EM: Constitutive expression of the CIRCADIAN CLOCK ASSOCIATED 1 (CCA1) gene disrupts circadian rhythms and suppresses its own expression. Cell 1998, 93:1207-1217.

41. Ito S, Matsushika A, Yamada H, Sato S, Kato T, Tabata S, Yamashino T, Mizuno T: Characterization of the APRR9 pseudo-response regulator belonging to the APRR1/TOC1 quintet in Arabidopsis thaliana. Plant Cell Physiol 2003, 44:1237-1245.

42. TIGR Arabidopsis thaliana Database http://www.tigr.org/tdb/e2k1/ ath1/

43. Rice Annotation Project Database [http://rapdb.dna.affrc.go.jp/]

44. JGI Sorghum bicolorv1.0 [http://genome.jgi-psf.org/Sorbi1/ Sorbi1.home.html]

45. Grape Genome Browser [http://www.genoscope.cns.fr/externe/ GenomeBrowser/Vitis/]

46. JGI Populus trichocarpa v1.1 [http://genome.jgi-psf.org/Poptr1 1/ Poptr1 1.home.html]

47. TCoffee [http://www.ebi.ac.uk/t-coffee/

48. TIGR Plant Transcript Assemblies [http://plantta.tigr.org/

49. Fgenesh+ [http://linux1.softberry.com/berry.phtml]

50. Jones DT, Taylor WR, Thornton JM: The rapid generation of mutation data matrices from protein sequences. Comput Appl Biosci 1992, 8:275-282.

51. Rzhetsky A, Nei M: A simple method for estimating and testing minimum evolution trees. Mol Biol Evol 1992, 9:945-967.

52. Felsenstein J: Confidence limits on phylogenies: an approach using the bootstrap. Evolution 1985, 39:783-791.

53. MEGA4 [http://www.megasoftware.net/index.html]

54. Tamura K, Dudley J, Nei M, Kumar S: MEGA4: Molecular Evolutionary Genetics Analysis (MEGA) software version 4.0. Mol Biol Evol 2007, 24:1596-1599

55. Gu X, Velden KVander: DIVERGE: phylogeny-based analysis for functional-structural divergence of a protein family. Bioinformatics 2002, 18:500-501.

56. DIVERGE 2.0 [http://xgu.zool.iastate.edu/]

57. Crooks GE, Hon G, Chandonia JM, Brenner SE: WebLogo: a sequence logo generator. Genome Res 2004, 14:1188-1190.

58. WebLogo 2.8.2 [http://weblogo.berkeley.edu/]

59. Lyons E, Freeling M: How to usefully compare homologous plant genes and chromosomes as DNA sequences. Plant J 2008, 53:661-673.

60. Harmer SL: The circadian system in higher plants. Annu Rev Plant Biol 2009, 60:357-377.

61. APGII [http://www.mobot.org/MOBOT/Research/APweb/welcome.html]

62. TIGR Plant Genomics [http://plantgenomics.tigr.org/

doi: $10.1186 / 1471-2148-10-126$

Cite this article as: Takata et al., Phylogenetic footprint of the plant clock system in angiosperms: evolutionary processes of Pseudo-Response Regulators BMC Evolutionary Biology 2010, 10:126 